\title{
Soliton states in mesoscopic two-band-superconducting cylinders
}

\author{
S. V. Kuplevakhsky, A. N. Omelyanchouk, and Y. S. Yerin* \\ B. I. Verkin Institute for Low Temperature Physics and Engineering, \\ National Academy of Sciences of Ukraine, and \\ 47 Lenin Ave., 61103 Kharkiv, UKRAINE
}

(Dated: November 5, 2018)

\begin{abstract}
In the framework of the Ginzburg-Landau approach, we present a self-consistent theory of specific soliton states in mesoscopic (thin-walled) two-band-superconducting cylinders in external parallel magnetic fields. Such states arise in the presence of "Josephson-type" interband coupling, when phase winding numbers are different for each component of the superconducting order parameter. We evaluate the Gibbs free energy of the sysyem up to second-order terms in a certain dimensionless parameter $\varepsilon \approx \frac{\mathcal{L}_{m}}{\mathcal{L}_{k}} \ll 1$, where $\mathcal{L}_{m}$ and $\mathcal{L}_{k}$ are the magnetic and kinetic inductance, respectively. We derive the complete set of exact soliton solutions. These solutions are thoroughly analyzed from the viewpoint of both local and global (thermodynamic) stability. In particular, we show that rotational-symmetry-breaking caused by the formation of solitons gives rise to a zero-frequency rotational mode. Although soliton states prove to be thermodynamically metastable, the minimal energy gap between the lowest-lying single-soliton states and thermodynamically stable zero-soliton states can be much smaller than the magnetic Gibbs free energy of the latter states, provided that intraband "penetration depths" differ substantially and interband coupling is weak. The results of our investigation may apply to a wide class of mesoscopic doubly-connected structures exhibiting two-band superconductivity.
\end{abstract}

PACS numbers: 74.20.De, 05.45.Yv

Keywords:

\section{INTRODUCTION}

The subject of this paper is a self-consistent theory of specific soliton states that were originally predicted in Ref. ${ }^{1}$ and reportedly observed experimentally $\underline{2}$ - Without any doubt, these states can be regarded as a hallmark of two-band superconductivity in mesoscopic doubly-connected samples.

Indeed, owing to the emergence of additional degrees of freedom of the order parameter, the nomenclature of topological objects in multiband superconductors is much richer than that in conventional single-band superconductors. In particular, Ginzburg-Landau equations describing two-band superconductivity in bulk samples admit topologically stable solutions (with one-dimensional singularities of the order parameter) that can be interpreted as vortices carrying fractional magnetic flux $\underline{3}^{-3}$ In the absence of any interband coupling, these vortices are accompanied by a circulating neutral superfow associated with gradients of the interband phase difference. In the presence of "Josephson-type" interband coupling, the neutral superflow generates static solitons of the sine-Gordon type. In contrast to traditional Abrikosov vortices in type-II superconductors, the energy per unit length of these composite topological defects diverges at spatial infinity: hence they are thermodynamically metastable and difficult to create in bulk samples.

However, solitons of the interband phase difference can exist by themselves in doubly-connected mesoscopic samples, when the formation of any magnetic vortices in the volume of the superconductor is prohibited energetically: $\underline{\underline{1}}$ Moreover, soliton states in this case can be induced by an externally applied magnetic field, which makes them a convenient object of investigation. Thus, experimental studies 2 of the magnetic response of mesoscopic two-band superconducting rings reveal certain nontrivial features that, according to the authors of Ref $\mathrm{f}^{2}$, can be attributed to the creation of metastable soliton states.

Our research is largely motivated by the absence in current literature of any quantitative theoretical analysis of this pronounced feature of two-band superconductivity. (Unfortunately, the arguments of Ref. $\frac{1}{1}$ and of the recent publications $\underline{\underline{4}}$ are mostly heuristic by nature.) Mathematically, the approach of this paper is based on a GinzburgLandau-type theory, which is a commonplace in theoretical studies of topological defects in two-band superconductors: see the next section. This means, of course, that we are restricted to the temperature range

$$
\frac{T_{c}-T}{T_{c}} \ll 1
$$

where $T_{c}$ is the critical temperature of the superconducting transition.

As to the physical object, we consider a two-band superconductor in the form of a straight, circular thin-walled cylinder, whose symmetry axis is the $z$ axis of cylindrical coordinates $(r, \varphi, z)$ (see Fig. 11). The constant external magnetic field $\mathbf{H}$ is applied along the symmetry axis: $\mathbf{H}=(0,0, H>0)$. The length of the generatrix of the wall of 


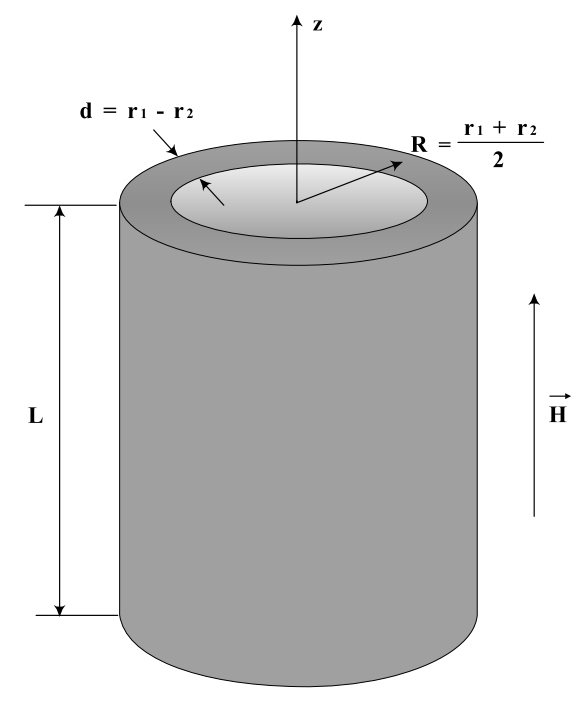

FIG. 1: The geometry of the problem (schematically). The parameters $L, R$ and $d$ obey conditions (2)-(5).

the cylinder satisfies the condition

$$
L \gg R \equiv \frac{r_{1}+r_{2}}{2},
$$

which allows us to neglect end effects. The wall thickness $d \equiv r_{2}-r_{1}$ and the average radius of the cylinder $R$ satisfy the following conditions:

$$
\begin{gathered}
d \ll \min \left\{\xi_{1}, \xi_{2}\right\}, \\
R \gg \max \left\{\xi_{1}, \xi_{2}\right\}, \\
R \gg \lambda, \\
\varepsilon \equiv \frac{d R}{2 \lambda^{2}} \ll 1,
\end{gathered}
$$

where $\xi_{1}$ and $\xi_{2}$ are the "coherence lengths" in bands 1 and 2, respectively, and $\lambda$ is the weak-field penetration depth. Exact definitions of $\xi_{1}, \xi_{2}$, and $\lambda$ will be given in the next section; however, the role of conditions (2)- (5) should be explained right now. Thus, condition (2) precludes the formation of any magnetic vortices in the wall of the cylinder. Condition (3) mainly simplifies mathematical consideration. In contrast, a combination of conditions (44) and (5) is of primary importance: taken together, these two conditions guarantee that self-induced magnetic fields are small and can be treated perturbatively. (This fact justifies the definition "mesoscopic cylinders" in the title of the paper.) Moreover, as will be shown, the dimensionless quantity $\varepsilon$ serves as a natural expansion parameter of the Gibbs free energy. In order to carry out a rigorous analysis of thermodynamic stability of soliton states, we will have to evaluate the Gibbs free energy exactly up to small terms of order $\varepsilon^{2}$, which implies the necessity of self-consistent evaluation of the vector potential up to first-order terms in $\varepsilon$.

We conclude the formulation of the problem by specifying boundary conditions for soliton states. Consider a two-component superconducting order parameter $\Psi=\left(\psi_{1}, \psi_{2}\right)$, where $\psi_{1}=\left|\psi_{1}\right| e^{i \phi_{1}}$ and $\psi_{2}=\left|\psi_{2}\right| e^{i \phi_{2}}$. The doubleconnectedness of the cylinder is accounted for by the condition of single-valuedness of $\Psi . \underline{5}$ In particular, this condition requires that

$$
\left|\psi_{1}\right|_{\varphi=0}=\left|\psi_{1}\right|_{\varphi=2 \pi},\left.\quad\left|\psi_{2}\right|\right|_{\varphi=0}=\left|\psi_{2}\right|_{\varphi=2 \pi} .
$$


As to the phases $\phi_{1}$ and $\phi_{2}$, the requirement is as follows:

$$
\oint_{\Gamma} \nabla \phi_{1} \cdot d \mathbf{l}=2 \pi n_{1}, \quad \oint_{\Gamma} \nabla \phi_{2} \cdot d \mathbf{l}=2 \pi n_{2}, \quad n_{1,2}=0, \pm 1, \pm 2, \ldots,
$$

where $\Gamma$ is an arbitrary closed continuous contour that lies inside the wall of the cylinder and encircles the opening. It should be emphasized that there are no a priori reasons for setting $n_{1}=n_{2}{ }^{6}$ As in the case of fractional magnetic vortices in bulk two-band superconductors, $\frac{3}{=}$ nontrivial topological states arise when $n_{1} \neq n_{2}$. In the presence of interband coupling, they are of the soliton type.

In Section II, we introduce the Gibbs free-energy functional of the system and analyze its basic properties. In Section III, we derive a self-consistent expression for the electromagnetic Gibbs free energy. Soliton solutions are derived and thoroughly discussed in Section IV. Finally, in Section V, we summarize the obtained results and make several concluding remarks. Appendices A and B contain details of some mathematical calculations skipped over in the main text. In Appendix C, we present several particular examples of soliton solutions.

\section{GIBBS FREE-ENERGY FUNCTIONAL}

We begin by defining the Gibbs free-energy functional of the system. In view of complete homogeneity along the $z$ axis and with the normal-state Gibbs free energy being subtracted, it takes the following form:

$$
\begin{gathered}
G\left[\Psi, \Psi^{*}, \mathbf{A} ; \mathbf{H}\right]=L \int_{\Sigma_{S}} d^{2} \mathbf{r}\left[\alpha_{1}\left|\psi_{1}\right|^{2}+\alpha_{2}\left|\psi_{2}\right|^{2}+\frac{\beta_{1}}{2}\left|\psi_{1}\right|^{4}+\frac{\beta_{2}}{2}\left|\psi_{2}\right|^{4}\right. \\
\left.+\frac{1}{2 m_{1}}\left|\left(-i \hbar \nabla-\frac{2 e}{c} \mathbf{A}\right) \psi_{1}\right|^{2}+\frac{1}{2 m_{2}}\left|\left(-i \hbar \nabla-\frac{2 e}{c} \mathbf{A}\right) \psi_{2}\right|^{2}-\gamma\left(\psi_{1} \psi_{2}^{*}+\psi_{1}^{*} \psi_{2}\right)\right] \\
+\frac{L}{8 \pi} \int_{\Sigma_{S}+\Sigma_{O}} d^{2} \mathbf{r}(\mathbf{h}-\mathbf{H})^{2} .
\end{gathered}
$$

Here, the coefficients $\beta_{1}$ and $\beta_{2}$ are positive constants, whereas $\alpha_{1}$ and $\alpha_{2}$ are temperature-dependent:

$$
\alpha_{1}=\alpha_{1}(T) \equiv a_{1}\left(T-T_{1}\right), \quad \alpha_{1}=\alpha_{2}(T) \equiv a_{2}\left(T-T_{2}\right), \quad a_{1}, a_{2}, T_{1}, T_{2}>0 .
$$

Moreover, the latter coefficients enter the definitions of the coherence lengths $\xi_{1}$ and $\xi_{2}$ :

$$
\xi_{1}=\frac{\hbar}{\sqrt{2 m_{1} \alpha_{1}}}, \quad \xi_{2}=\frac{\hbar}{\sqrt{2 m_{2} \alpha_{2}}} .
$$

The electron charge in (8) is $e<0$; the total vector potential $\mathbf{A}$ defines the local magnetic field $\mathbf{h}$ :

$$
\mathbf{h}=\nabla \times \mathbf{A}, \quad \mathbf{h}=(0,0, h), \quad h=h(r) .
$$

The parameter of interband coupling, $\gamma$, may have either sign. Two-dimensional integration in the plane $(r, \varphi)$ is carried out over the cross-section of the superconductor $\left(\Sigma_{S}\right)$ in the square-bracketed terms, and over the crosssections of the superconductor and of the opening $\left(\Sigma_{S}+\Sigma_{O}\right)$ in the last (magnetic) term.

A microscopic derivation of free-energy functionals of the type (8) was given in Ref $\underline{7}$ for the case of clean twoband superconductors in the limit of small interband coupling. Free-energy functionals of this type are employed in theoretical studies of different aspects of two-band superconductivity, such as, e.g., topological defects,,$\frac{1,3.8}{\Perp}$ currentcarrying states,,$\underline{\underline{9}}$ the Little-Parks effect,,$\underline{10}$ surface energy, $\stackrel{11}{\underline{e}}$ etc. It should be additionally noted that, for our specific geometry and $\gamma>0$, the functional (8) also applies to the description of a composite system consisting of two thin coaxial cylindrical films of single-band superconductors, Josephson coupled via a parallel insulating layer, 12 which is exactly the experimental set-up of Ref $\stackrel{2}{2}$.

To obtain the actual (observable) Gibbs free energy, one has to minimize (8) with respect to $\Psi, \Psi^{*}$ and $\mathbf{A}$ under appropriate boundary conditions; however, substantial simplifications can be made already at this stage. First, we notice that, by the symmetry of the problem, the amplitudes $\left|\psi_{1}\right|$ and $\left|\psi_{2}\right|$ do not depend on $\varphi$. Moreover, they cannot depend on $r$, either. Indeed, by virtue of condition (2), any radial variations of $\left|\psi_{1}\right|$ and $\left|\psi_{2}\right|$ would give rise to free-energy terms that are by the factors $\frac{\xi_{1}^{2}}{d^{2}} \gg 1$ and $\frac{\xi_{2}^{2}}{d^{2}} \gg 1$ larger than the first and the second terms in (8), respectively, which is energetically unfavorable $\stackrel{13}{=}$ As a result, in equilibrium, the magnitudes $\left|\psi_{1}\right|$ and $\left|\psi_{2}\right|$ are functions of $T$ and $H$ only. 
Consider now the kinetic-energy terms [the first two terms in the second line of (8)]. The ratio of these terms to the first and the second terms in (8), respectively, is at most of order $\frac{\xi_{1}^{2}}{R^{2}}\left(\frac{\Phi_{H}}{\Phi_{0}}\right)^{2}$ and $\frac{\xi_{2}^{2}}{R^{2}}\left(\frac{\Phi_{H}}{\Phi_{0}}\right)^{2}$, where $\Phi_{H}$ is the external flux, and

$$
\Phi_{0}=\frac{\pi \hbar c}{|e|}
$$

is the flux quantum. Owing to condition (3), for sufficiently weak external fields, $\frac{\xi_{1}^{2}}{R^{2}}\left(\frac{\Phi_{H}}{\Phi_{0}}\right)^{2}, \frac{\xi_{2}^{2}}{R^{2}}\left(\frac{\Phi_{H}}{\Phi_{0}}\right)^{2} \ll 1$. (Compare with the consideration of flux quantization in singe-band-superconducting cylinders. $\left.{ }^{14}\right)$ In this field range, we can set $\left|\psi_{1}\right|=\left|\psi_{1}\right|_{0}$ and $\left|\psi_{2}\right|=\left|\psi_{2}\right|_{0}$, where $\left|\psi_{1}\right|_{0}$ and $\left|\psi_{2}\right|_{0}$ satisfy the equilibrium conditions for an unperturbed two-band superconductor:

$$
\begin{aligned}
& \alpha_{1}\left|\psi_{1}\right|_{0}+\beta_{1}\left|\psi_{1}\right|_{0}^{3}-|\gamma|\left|\psi_{2}\right|_{0}=0 \\
& \alpha_{2}\left|\psi_{2}\right|_{0}+\beta_{2}\left|\psi_{2}\right|_{0}^{3}-|\gamma|\left|\psi_{1}\right|_{0}=0
\end{aligned}
$$

One can readily obtain a good approximate solution to (13) ${ }^{15}$ However, it is of no interest in the context of the soliton

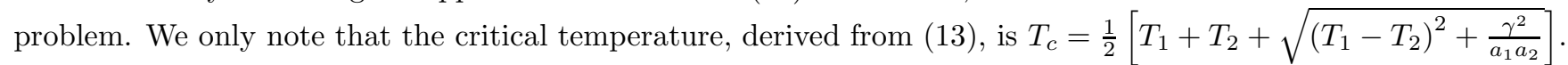

In light of these simplifications, it is reasonable to consider the weak-field penetration depth 15

$$
\lambda=\frac{c}{4 \sqrt{\pi}|e|} \sqrt{\frac{m_{1} m_{2}}{m_{2}\left|\psi_{1}\right|_{0}^{2}+m_{1}\left|\psi_{2}\right|_{0}^{2}}}
$$

and to define intraband "penetration depths"11

$$
\lambda_{1}=\frac{c}{4 \sqrt{\pi}|e|} \frac{\sqrt{m_{1}}}{\left|\psi_{1}\right|_{0}}, \quad \lambda_{2}=\frac{c}{4 \sqrt{\pi}|e|} \frac{\sqrt{m_{2}}}{\left|\psi_{2}\right|_{0}} ; \quad \lambda_{1}^{-2}+\lambda_{2}^{-2}=\lambda^{-2} .
$$

(For the above-mentioned composite, Josephson-coupled system, the quantities $\lambda_{1}$ and $\lambda_{2}$ have direct physical meaning.) We also introduce new, functionally independent phase variables $\not \phi$ and $\chi^{: 9,10}$

$$
\begin{gathered}
\phi=\varphi_{1}-\varphi_{2}, \\
\chi=c_{1} \varphi_{1}+c_{2} \varphi_{2} ; \quad c_{1} \equiv\left(\lambda \lambda_{1}^{-1}\right)^{2}, \quad c_{2} \equiv\left(\lambda \lambda_{2}^{-1}\right)^{2}, \quad c_{1}+c_{2}=1 .
\end{gathered}
$$

Using definitions (12) and (14)-(17), we obtain the reduced Gibbs free-energy functional in the following form:

$$
G[\phi, \chi, \mathbf{A} ; \mathbf{H}]=F_{S 0}+G_{e m}[\chi, \mathbf{A} ; \mathbf{H}]+F_{s o l}[\phi] .
$$

Here, the first term is the free energy of the unperturbed superconducting cylinder $:^{10}$

$$
\begin{gathered}
F_{S 0}=V_{S}\left(\alpha_{1}\left|\psi_{1}\right|_{0}^{2}+\alpha_{2}\left|\psi_{2}\right|_{0}^{2}+\frac{\beta_{1}}{2}\left|\psi_{1}\right|_{0}^{4}+\frac{\beta_{2}}{2}\left|\psi_{2}\right|_{0}^{4}-2|\gamma|\left|\psi_{1}\right|_{0}\left|\psi_{2}\right|_{0}\right) ; \\
V_{S} \equiv 2 \pi R L d .
\end{gathered}
$$

The second term is the electromagnetic Gibbs free-energy functional:

$$
G_{e m}[\chi, \mathbf{A} ; \mathbf{H}]=\frac{\Phi_{0}^{2}}{32 \pi^{3}} \frac{L}{\lambda^{2}} \int_{\Sigma_{S}} d^{2} \mathbf{r}\left(\nabla \chi-\frac{2 e}{\hbar c} \mathbf{A}\right)^{2}+\frac{L}{8 \pi} \int_{\Sigma_{S}+\Sigma_{O}} d^{2} \mathbf{r}(\mathbf{h}-\mathbf{H})^{2},
$$

with the first term on the right-hand side of (20) being the kinetic-energy functional of the supercurrent. Finally, the last term in (18) is

$$
\begin{gathered}
F_{\text {sol }}[\phi]=\frac{\Phi_{0}^{2}}{32 \pi^{3}} \frac{L}{\lambda^{2}} c_{1} c_{2} \int_{\Sigma_{S}} d^{2} \mathbf{r}\left[(\nabla \phi)^{2}+\frac{2}{l^{2}}(1-\operatorname{sgn} \gamma \cos \phi)\right] \\
l^{2} \equiv \frac{\Phi_{0}^{2}}{32 \pi^{3}} \frac{1}{\lambda^{2}} \frac{c_{1} c_{2}}{|\gamma|\left|\psi_{1}\right|_{0}\left|\psi_{2}\right|_{0}},
\end{gathered}
$$

where $\operatorname{sgn} x$ is the sign function. The term (21) should be interpreted as the soliton self-energy functional. Indeed,

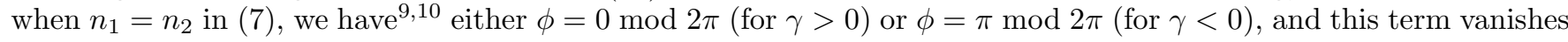
identically.

Our task now is to minimize (18) with respect to $\phi, \chi$ and $\mathbf{A}$. As the phase variable $\phi$ is not coupled to the vector potential $\mathbf{A}$, this procedure can be performed in two separate steps. 


\section{ELECTROMAGNETIC GIBBS FREE ENERGY}

The minimization of the electromagnetic functional (20) reduces to evaluation of the stationarity condition $\delta G_{e m}=$ 0 , or

$$
\frac{\delta G_{e m}}{\delta \mathbf{A}}=0, \quad \frac{\delta G_{e m}}{\delta \chi}=0
$$

Indeed, in view of quadratic nature of (20), solutions to (22) are automatically minimizers of this functional (i.e., $\delta^{2} G_{e m}>0$ at these solutions).

Variation with respect to A yields Ampère's law

$$
\begin{gathered}
\nabla \times \nabla \times \mathbf{A}=0, \quad r \in\left(0, r_{1}\right) \\
\nabla \times \nabla \times \mathbf{A}=\frac{4 \pi}{c} \mathbf{j}, \quad r \in\left(r_{1}, r_{2}\right),
\end{gathered}
$$

with

$$
\mathbf{j}=-\frac{c}{4 \pi \lambda^{2}}\left(\frac{\Phi_{0}}{2 \pi} \nabla \chi+\mathbf{A}\right)
$$

being the supercurrent density $[\mathbf{j}=(0, j, 0)$ by symmetry], and the boundary condition

$$
\left.\mathbf{h}\right|_{r=r_{2}} \equiv \nabla \times\left.\mathbf{A}\right|_{r=r_{2}}=\mathbf{H}
$$

(This boundary condition should, of course, be complemented by the conditions of continuity of $\mathbf{A}$ and $\mathbf{h}$ at $r=r_{2}$ and the condition of regularity of $\mathbf{A}$ at the origin.) Variation with respect to $\chi$, under the condition of single-valuedness of variations $\delta \chi$, just yields the current-conservation law

$$
\nabla \mathbf{j}=0
$$

and the single-valuedness condition

$$
\left.j\right|_{\varphi=0}=\left.j\right|_{\varphi=2 \pi}
$$

[This boundary condition should be complemented by a condition on $\chi$ resulting from (17).]

The problem of finding $\mathbf{A}$ and $\chi$ is still sub-definite, because we have not so far fixed the gauge. As the $z$ component of the vector potential drops out of the definition of $\mathbf{h}$ [see (11)], it is equal to an arbitrary constant, and we set $A_{z} \equiv 0$. The $r$ component of the vector potential can be eliminated by the gauge transformation

$$
\mathbf{A} \rightarrow \mathbf{A}-\nabla \int_{0}^{r} A_{r}\left(r^{\prime}, \varphi\right) d r^{\prime}, \quad \chi \rightarrow \chi+\frac{2 \pi}{\Phi_{0}} \int_{0}^{r} A_{r}\left(r^{\prime}, \varphi\right) d r^{\prime}
$$

In this particular gauge,

$$
\begin{gathered}
\mathbf{A}=(0, A, 0), \quad A=A(r) ; \\
h=\frac{1}{r} \frac{d}{d r}(r A),
\end{gathered}
$$

and $\chi$ does not depend on $r\left(j_{r} \equiv 0\right)$. Using (7), (17), (27) and (28), we arrive at a well-posed boundary-value problem,

$$
\begin{gathered}
\frac{d^{2} \chi}{d \varphi^{2}}=0, \quad \varphi \in(0,2 \pi) \\
\chi(2 \pi)=\chi(0)+2 \pi\left(n_{1} c_{1}+n_{2} c_{2}\right), \quad \frac{d \chi}{d \varphi}(2 \pi)=\frac{d \chi}{d \varphi}(0),
\end{gathered}
$$

whose solution is

$$
\chi(\varphi)=\left(n_{1} c_{1}+n_{2} c_{2}\right) \varphi+\varphi_{0},
$$


with $\varphi_{0}$ being an arbitrary constant.

The boundary-value problem for the vector potential now takes the form

$$
\begin{gathered}
\frac{d}{d r}\left[\frac{1}{r} \frac{d}{d r}(r A)\right]=0, \quad r \in\left(0, r_{1}\right) ; \\
\frac{d}{d r}\left[\frac{1}{r} \frac{d}{d r}(r A)\right]=\frac{1}{\lambda^{2}}\left[A+\frac{\Phi_{0}}{2 \pi} q\left(n_{1}, n_{2}\right)\right], \quad r \in\left(r_{1}, r_{2}\right) ; \\
q\left(n_{1}, n_{2}\right) \equiv n_{1} c_{1}+n_{2} c_{2} ; \\
\left|A \|_{r=0}<\infty, A\right|_{r=r_{1}-0}=\left.A\right|_{r=r_{1}+0},\left.\frac{1}{r} \frac{d}{d r}(r A)\right|_{r=r_{1}-0}=\left.\frac{1}{r} \frac{d}{d r}(r A)\right|_{r=r_{1}+0}, \\
\left.\frac{1}{r} \frac{d}{d r}(r A)\right|_{r=r_{2}}=H .
\end{gathered}
$$

This boundary-value problem admits an exact solution: it is presented in Appendix A. However, to obtain a secondorder expansion of the electromagnetic Gibbs free energy in terms of the small parameter $\varepsilon$ (see Introduction), we need only first-order expansions of $A$ and $h$. They are as follows:

$$
\begin{aligned}
A(r) & =\frac{r}{2} H-\frac{r}{2}\left[\frac{\Phi_{0}}{\pi r_{1}^{2}} q\left(n_{1}, n_{2}\right)+H\right] \varepsilon, \quad r \in\left[0, r_{1}\right] ; \\
& =\frac{r}{2} H-\frac{r_{1}}{2}\left[\frac{\Phi_{0}}{\pi r_{1}^{2}} q\left(n_{1}, n_{2}\right)+H\right] \varepsilon, \quad r \in\left(r_{1}, r_{2}\right] ; \\
h(r)= & H-\left[\frac{\Phi_{0}}{\pi r_{1}^{2}} q\left(n_{1}, n_{2}\right)+H\right] \varepsilon, \quad r \in\left[0, r_{1}\right] ; \\
= & H-\frac{r_{2}-r}{r_{2}-r_{1}}\left[\frac{\Phi_{0}}{\pi r_{1}^{2}} q\left(n_{1}, n_{2}\right)+H\right] \varepsilon, \quad r \in\left(r_{1}, r_{2}\right] .
\end{aligned}
$$

[The fact that expressions (33) and (34) on the interval $\left(r_{1}, r_{2}\right)$ are not related to each other by equation (30) should not cause any confusion: to ensure the fulfillment of (30), we would have to continue the expansion of (A4) up to small terms of order $\frac{d}{R}$ and $\varepsilon \frac{d}{R}$.]

Bearing in mind that in integral physical quantities any difference between $r_{1}, r_{2}$ and $R$ should be neglected [see (A8)], by use of equations (34) and (24) we immediately obtain expressions for the total flux $\Phi=\int_{\Sigma_{O}} h d^{2} \mathbf{r}$ and the total supercurrent $J=L \int_{r_{1}}^{r_{2}} j d r$ :

$$
\begin{gathered}
\Phi=\Phi_{H}+\Phi_{i}, \quad \Phi_{H}=\pi R^{2} H, \quad \Phi_{i}=-\left[\Phi_{0} q\left(n_{1}, n_{2}\right)+\Phi_{H}\right] \varepsilon \\
J=\frac{c}{\mathcal{L}_{m}} \Phi_{i}, \quad \mathcal{L}_{m}=\frac{4 \pi^{2} R^{2}}{L} .
\end{gathered}
$$

Here, $\Phi_{H}$ and $\Phi_{i}$ are the external and self-induced flux, respectively; $\mathcal{L}_{m}$ is the magnetic inductance of the cylinder (or self-inductance) $\underline{\underline{16}}$

On substitution of relations (31), (33) and (34) into (20), we get the electromagnetic Gibbs free energy of the state parameterized by topological numbers $n_{1}$ and $n_{2}$ :

$$
\begin{aligned}
G_{e m}\left(n_{1}, n_{2} ; H\right) & =\frac{\left[\Phi_{0} q\left(n_{1}, n_{2}\right)+\Phi_{H}\right]^{2}}{2 \mathcal{L}_{m}} \varepsilon(1-2 \varepsilon)+\frac{\left[\Phi_{0} q\left(n_{1}, n_{2}\right)+\Phi_{H}\right]^{2}}{2 \mathcal{L}_{m}} \varepsilon^{2} \\
= & \frac{\Phi_{0}^{2} \varepsilon}{2 \mathcal{L}_{m}}\left[q\left(n_{1}, n_{2}\right)+f_{H}\right]^{2}(1-\varepsilon), \quad f_{H} \equiv \frac{\Phi_{H}}{\Phi_{0}} .
\end{aligned}
$$

(Note that the first term in the first line of this equation is the kinetic energy of the supercurrent, whereas the second term in the same line is the magnetic Gibbs free energy.) 
To facilitate an analysis of soliton states, we should transform (37) to a more convenient form. First, instead of parameterization by $n_{1}$ and $n_{2}$, we introduce parameterization by $n_{1}$ and the soliton number $n=n_{1}-n_{2}$. From now on, we assume (without any loss of generality) that $0<c_{2} \leq c_{1}<1$. Relation (37) is rewritten as follows:

$$
G_{e m}\left(n_{1}, n ; f_{H}\right)=\frac{\Phi_{0}^{2} \varepsilon}{2 \mathcal{L}_{m}}\left(f_{H}+n_{1}-n c_{2}\right)^{2}(1-\varepsilon) .
$$

From a thermodynamic point of view, of interest is the minimum of (38) for given $|n|=0,1,2, \ldots$. Therefore, relation (38) should be minimized with respect to $n_{1}$ and sng $n$. With this in mind, we introduce two discontinuous functions, a step function $m(x)$ and a periodic function $\theta(x)$, via the definitions

$$
m(x)=\left\{\begin{array}{c}
{[x], \quad 0 \leq\{x\} \leq \frac{1}{2}} \\
{[x]+1, \quad \frac{1}{2}<\{x\}<1,}
\end{array}\right.
$$

and

$$
\theta(x)=\left\{\begin{array}{c}
\{x\}, \quad 0 \leq\{x\} \leq \frac{1}{2} \\
-1+\{x\}, \quad \frac{1}{2}<\{x\}<1
\end{array}\right.
$$

where $[x]$ and $\{x\}$ are the integer and fractional parts of $x$, respectively. Given that $f_{H}$ and $n c_{2}$ can now be represented as $f_{H}=m\left(f_{H}\right)+\theta\left(f_{H}\right)$ and $n c_{2}=\operatorname{sgn} n\left[m\left(|n| c_{2}\right)+\theta\left(|n| c_{2}\right)\right]$, respectively, the result of the minimization is

$$
\begin{gathered}
G_{e m}\left(|n| ; f_{H}\right)=\min _{n_{1}, \mathrm{sgn} n} G_{e m}\left(n_{1}, n ; f_{H}\right) \\
=G_{e m}\left(-m\left(f_{H}\right)+m\left(|n| c_{2}\right) \operatorname{sgn} \theta\left(f_{H}\right) \operatorname{sgn} \theta\left(|n| c_{2}\right),|n| \operatorname{sgn} \theta\left(f_{H}\right) \operatorname{sgn} \theta\left(|n| c_{2}\right) ; f_{H}\right) \\
=\frac{\Phi_{0}^{2} \varepsilon}{2 \mathcal{L}_{m}}\left[\left|\theta\left(f_{H}\right)\right|-\left|\theta\left(|n| c_{2}\right)\right|\right]^{2}(1-\varepsilon) .
\end{gathered}
$$

\section{SOLITON STATES}

\section{A. Soliton self-energy}

The variation of (21) with respect to $\phi$, under the condition of single-valuedness of variations $\delta \phi$, yields a static two-dimensional sine-Gordon equation in polar coordinates,

$$
\frac{1}{r^{2}} \frac{\partial^{2} \phi}{\partial \varphi^{2}}+\frac{1}{r} \frac{\partial}{\partial r}\left(r \frac{\partial \phi}{\partial r}\right)=\frac{\operatorname{sgn} \gamma}{l^{2}} \sin \phi, \quad r \in\left(r_{1}, r_{2}\right), \quad \varphi \in(0,2 \pi)
$$

and the boundary conditions

$$
\left.\frac{1}{r} \frac{\partial \phi}{\partial \varphi}\right|_{\varphi=0}=\left.\frac{1}{r} \frac{\partial \phi}{\partial \varphi}\right|_{\varphi=2 \pi} ;\left.\quad \frac{\partial \phi}{\partial r}\right|_{r=r_{1}}=\left.\frac{\partial \phi}{\partial r}\right|_{r=r_{2}}=0 .
$$

[These boundary conditions should, of course, be complemented by a condition on $\phi$ resulting from (77).]

However, equation (42), in its exact form, by far exceeds the accuracy of our calculations in the previous section [see expressions (33), (34) and relations (48)]. Discarding in (42) terms of order $\frac{d}{R}$ and $\frac{d^{2}}{R^{2}}$, we arrive at a two-dimensional sine-Gordon equation in "Cartesian coordinates":

$$
\frac{\partial^{2} \phi}{\partial \varphi^{2}}+\frac{\partial^{2} \phi}{\partial \rho^{2}}=\frac{R^{2} \operatorname{sgn} \gamma}{l^{2}} \sin \phi, \quad \rho \equiv \frac{r}{R} .
$$

Solutions to (44), minimizing the functional (21), should not depend on $\rho$ for symmetry reasons [which, of course, is compatible with boundary conditions (43)].

Thus, the phase $\phi=\phi(\varphi)$ satisfies the following boundary-value problem:

$$
\begin{gathered}
\frac{d^{2} \phi}{d \varphi^{2}}=\frac{R^{2} \operatorname{sgn} \gamma}{l^{2}} \sin \phi, \quad \varphi \in(0,2 \pi) ; \\
\phi(2 \pi)=\phi(0)+2 \pi n \quad(n= \pm 1, \pm 2, \ldots), \quad \frac{d \phi}{d \varphi}(2 \pi)=\frac{d \phi}{d \varphi}(0) .
\end{gathered}
$$


The solution of (45) is straightforward: ${ }^{17}$

$$
\phi_{n}(\varphi)=\frac{(1+\operatorname{sgn} \gamma) \pi}{2}+2 \operatorname{am}\left(\frac{n K\left(k_{n}\right)}{\pi}\left(\varphi-\varphi_{n 0}\right), k_{n}\right),
$$

where am $u$ is the elliptic amplitude, ${ }^{18} K(k)$ is the complete elliptic integral of the first kind, $\stackrel{18}{\varphi_{n 0}}$ are arbitrary constants, and $k_{n}(n= \pm 1, \pm 2, \ldots)$ satisfy the equations

$$
|n| k_{n} K\left(k_{n}\right)=\frac{\pi R}{l}, \quad n= \pm 1, \pm 2, \ldots
$$

Particular examples of solutions (46) that possess asymptotics in terms of elementary functions are relegated to Appendix C. Nonetheless, the very special class of exact elementary solutions is worth being presented here: namely, the non-soliton nontrivial topological solutions corresponding to the physical case of the absence of interband coupling $(|\gamma|=0)$. These solutions can be obtained from (46) by the limit procedure

$$
k_{n} \rightarrow 0, \quad n= \pm 1, \pm 2, \ldots
$$

and they have the general form

$$
\phi_{n}(\varphi)=n \varphi+\varphi_{0}
$$

They are necessarily minimizers of (21) [i.e., at these solutions $\delta^{2} F_{\text {sol }}>0$, because the functional (21) is quadratic in the case $|\gamma|=0]$, and their self-energy is

$$
F_{\text {sol }}^{(0)}(n)=\lim _{k_{n} \rightarrow 0} F_{\text {sol }}(n)=\frac{\Phi_{0}^{2} \varepsilon}{2 \mathcal{L}_{m}}|n|^{2} c_{1} c_{2}
$$

If $|\gamma| \neq 0$, the functional (21) is non-quadratic, and we should analyze the second variation of (21) in more detail. To this end, 17,19 we turn to the Sturm-Liouville problem

$$
\begin{gathered}
-\frac{d^{2} \psi}{d \varphi^{2}}+\cos \not \phi_{n} \psi=\mu \psi, \quad \varphi \in(0,2 \pi) ; \\
\psi(0)=\psi(2 \pi), \quad \frac{d \psi}{d \varphi}(0)=\frac{d \psi}{d \varphi}(2 \pi),
\end{gathered}
$$

where $\not \phi_{n}$ is a given solution from the set (46). As shown in Refs, 17,19 ,

$$
\left.\delta^{2} F_{s o l}\right|_{\phi=\phi_{n}} \geq \mu_{0} \int_{0}^{2 \pi}\left|\delta \phi_{n}\right|^{2} d \varphi
$$

where $\mu_{0}$ is lowest eigenvalue of the problem (51). In our case, both $\mu_{0}$ and the corresponding eigenfunction $\psi_{0}$ can be readily found:

$$
\mu_{0}=0, \quad \psi_{0}=\text { const } \operatorname{dn}\left(\frac{n K\left(k_{n}\right)}{\pi}\left(\varphi-\varphi_{n 0}\right), k_{n}\right)
$$

where $\operatorname{dn} u=\frac{d \operatorname{am} u}{d u} \stackrel{18}{1}$ This means that $\left.\delta^{2} F_{\text {sol }}\right|_{\phi=\phi_{n}} \geq 0$, and soliton states turn out to be indifferently stable states. Indeed, the zero value of $\mu_{0}$ should be attributed to the existence of a zero-frequency "rotational mode" (by analogy with the well-known ${ }^{20}$ translational mode in quantum field theories) that restores rotational symmetry broken by the formation of solitons. To prove this, consider a small variation of $\phi_{n}$ induced by a small variation of the constant of integration $\varphi_{n 0}$ :

$$
\phi_{n}(\varphi) \rightarrow \phi_{n}\left(\varphi+\frac{\pi}{n K} \alpha\right) \approx \phi_{n}(\varphi)+\alpha \operatorname{dn}\left(\frac{n K\left(k_{n}\right)}{\pi}\left(\varphi-\varphi_{n 0}\right), k_{n}\right), \quad|\alpha| \ll 1 .
$$

From the above, we see that $\delta \phi_{n} \propto \psi_{0}$.

Now that local stability of soliton solutions is established, we proceed with a discussion of soliton self-energy. It is obtained by the substitution of solutions (46) into (21) and has the form

$$
F_{\text {sol }}(n)=\frac{\Phi_{0}^{2} \varepsilon}{\mathcal{L}_{m}} \frac{2|n|^{2}}{\pi^{2}} c_{1} c_{2} K\left(k_{n}\right)\left[2 E\left(k_{n}\right)-\left(1-k_{n}^{2}\right) K\left(k_{n}\right)\right],
$$


where $E(k)$ is the complete elliptic integral of the second kind $\underline{18}$

First, we note that the constants of integration $\varphi_{n 0}$ that figure in (46) drop out of the right-hand side of (52), as they should. The self-energy does not depend on the sign of $\gamma$ and of $n$, either. By considering (formally) $|n|$ as a continuous variable, we get

$$
\frac{\partial F_{\text {sol }}(n)}{\partial|n|}=\frac{\Phi_{0}^{2} \varepsilon}{\mathcal{L}_{m}} \frac{4|n| R}{\pi l} c_{1} c_{2} E\left(k_{n}\right)>0
$$

which means that $F_{\text {sol }}(n)$ increases monotonically with an increase in $|n|$, as could be expected. However, in contrast to the case $|\gamma|=0$ [see (50)], the growth of $F_{\text {sol }}(n)$ is slower than $|n|^{2}$, because

$$
\frac{\partial}{\partial|n|}\left[\frac{F_{\text {sol }}(n)}{|n|^{2}}\right]=-\frac{\Phi_{0}^{2} \varepsilon}{\mathcal{L}_{m}} \frac{4 R}{\pi|n|^{2} l} c_{1} c_{2} \frac{E\left(k_{n}\right)-\left(1-k_{n}^{2}\right) K\left(k_{n}\right)}{k_{n}}<0 .
$$

Given that

$$
\frac{\partial F_{\text {sol }}(n)}{\partial k_{n}}=\frac{\Phi_{0}^{2} \varepsilon}{\mathcal{L}_{m}} \frac{4|n|^{2}}{\pi^{2}} c_{1} c_{2} \frac{E\left(k_{n}\right)\left[E\left(k_{n}\right)-\left(1-k_{n}^{2}\right) K\left(k_{n}\right)\right]}{k_{n}\left(1-k_{n}^{2}\right)}>0, \quad k_{n} \in(0,1),
$$

the self-energy increases monotonically with an increase in $k_{n}$ on the whole interval $(0,1)$. The minimal value of (52) is achieved at $k_{n}=0$ and is given by (50). In view of the relation

$$
\frac{\partial k_{n}}{\partial\left(\frac{l}{R}\right)}=-\frac{\pi R^{2}}{|n|^{2} l^{2}} \frac{1-k_{n}^{2}}{E\left(k_{n}\right)}<0
$$

the self-energy decreases monotonically with an increase in $\frac{l}{R} \in(0, \infty)$ (for a given $\left.|n|\right)$. [In other words, $F_{\text {sol }}$ is an increasing function of the interband coupling parameter $|\gamma|$ : see the definition of $l$ in (21).]

\section{B. Thermodynamic metastability}

According to (18), (41) and (52) the minimal Gibbs free energy of soliton states with a given $|n|$ in the field $H$ can be represented as follows:

$$
\begin{gathered}
G\left(|n| ; f_{H}\right)=F_{0}+\frac{\Phi_{0}^{2} \varepsilon}{2 \mathcal{L}_{m}}\left[\left[\left|\theta\left(f_{H}\right)\right|-\left|\theta\left(|n| c_{2}\right)\right|\right]^{2}(1-\varepsilon)\right. \\
\left.+\frac{4|n|^{2}}{\pi^{2}} c_{1} c_{2} K\left(k_{n}\right)\left[2 E\left(k_{n}\right)-\left(1-k_{n}^{2}\right) K\left(k_{n}\right)\right]\right] .
\end{gathered}
$$

To analyze thermodynamic stability of soliton solutions, we should compare expression (53) for $|n| \geq 1$ with the Gibbs free energy of the states with $|n|=0$ :

$$
G\left(0 ; f_{H}\right)=F_{0}+\frac{\Phi_{0}^{2} \varepsilon}{2 \mathcal{L}_{m}}\left|\theta\left(f_{H}\right)\right|^{2}(1-\varepsilon) .
$$

With this in mind, we first note that, for $|n| \geq 1$, the energy $G\left(|n| ; f_{H}\right)$ increases monotonically with an increase in $|n|$ : see Appendix B for a proof. [Contrary to what may seem, this fact is by no means obvious, because the electromagnetic term in (53) may decrease with an increase in $|n|$.$] Furthermore, since expression (50) provides the$ greatest lower bound for soliton self-energies, we can restrict ourselves to the case $|n|=1$ and $k_{n}=0$. Bearing in mind that $c_{2} \in\left(0, \frac{1}{2}\right]$ by assumption (see the end of Section III), we arrive at the following important inequalities:

$$
\begin{gathered}
\Delta G\left(1 ; f_{H}\right) \equiv G\left(1 ; f_{H}\right)-G\left(0 ; f_{H}\right) \\
\geq \Delta G^{(0)}\left(1 ; f_{H}\right) \equiv \lim _{k_{n} \rightarrow 0} G\left(1 ; f_{H}\right)-G\left(0 ; f_{H}\right) \\
=\frac{\Phi_{0}^{2} \varepsilon}{2 \mathcal{L}_{m}} c_{2}\left[1-c_{2} \varepsilon-2\left|\theta\left(f_{H}\right)\right|(1-\varepsilon)\right]>0 .
\end{gathered}
$$



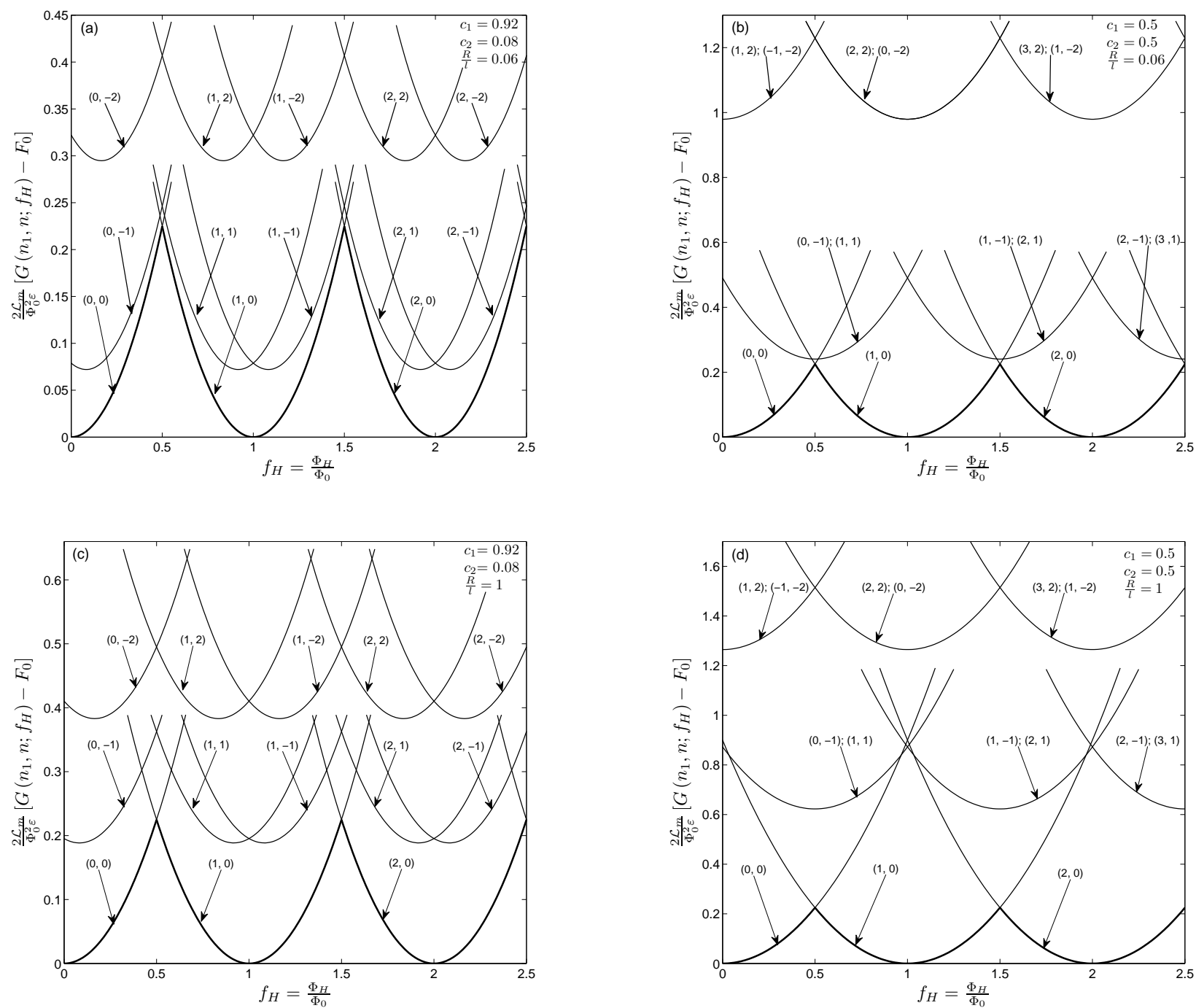

FIG. 2: Gibbs free energy of several different topological states $\left(n_{1}, n\right)(|n|=0,1,2)$ for typical values of the parameters $c_{1}, c_{2}$, and $\frac{R}{l}$. Note double degeneracy of soliton states in the case $c_{1}=c_{2}=\frac{1}{2}$ and see the text for further explanations.

The above inequalities clearly demonstrate thermodynamic metastability of soliton states and bring to light certain subtle physical points. In particular,

$$
\max _{f_{H}} \Delta G\left(1 ; f_{H}\right)=\left.\Delta G\left(1 ; f_{H}\right)\right|_{f_{H}=p} \geq \frac{\Phi_{0}^{2} \varepsilon}{2 \mathcal{L}_{m}} c_{2}\left(1-c_{2} \varepsilon\right), \quad p=0,1,2, \ldots
$$

In contrast,

$$
\min _{f_{H}} \Delta G\left(1 ; f_{H}\right)=\left.\Delta G\left(1 ; f_{H}\right)\right|_{f_{H}=p+\frac{1}{2}} \geq \frac{\Phi_{0}^{2} \varepsilon^{2}}{2 \mathcal{L}_{m}} c_{1} c_{2}, \quad p=0,1,2, \ldots,
$$

which shows that $\min _{f_{H}} \Delta G\left(1 ; f_{H}\right)$ can be much smaller than the magnetic Gibbs free energy of the zero-soliton states [see (37)], provided that $0<c_{2} \ll c_{1}<1$ [i.e., when $\lambda_{1} \ll \lambda_{2}<\infty$, see (17)] and $\frac{l}{R} \gg 1$ (weak interband coupling).

In Fig. 2] we plot the Gibbs free energy of several different topological states $\left(n_{1}, n\right)(|n|=0,1,2)$. Thermodynamically stable zero-soliton states are denoted by thick solid lines. Minima of the Gibbs free energy of soliton states 
represent the soliton self-energy and occur when the self-induced flux $\Phi_{i}$ compensates for the external flux $\Phi_{H}$, i.e., when

$$
f_{H}+n_{1}-n c_{2}=0
$$

[see relations (35), (38) and (41)]. In the very special case, when $c_{2}=c_{1}=\frac{1}{2}$ and $n=2 n_{1}$, no flux is induced $\left(\Phi_{i}=0\right)$, and minima of the soliton Gibbs free energy occur at $H=0$.

\section{SUMMARY AND CONCLUSIONS}

Summarizing, we have presented (in the framework of the Ginzburg-Landau approach) a self-consistent theory of specific soliton states that constitute a distinctive feature of two-band superconductivity in mesoscopic multiplyconnected samples. Although our mathematical consideration concerns the concrete geometry of Fig. 1, the final results can be expressed in terms of the magnetic and kinetic inductance (see Ref. ${ }^{16}$ ) and, therefore, should apply to a much wider class of structures. This allows us to make several generalizing remarks.

As the predicted fractional magnetic vortices in bulk two-band superconductors,$\frac{3}{\underline{3}}$ the soliton states considered here prove to be thermodynamically metastable. However, the minimal energy gap between the lowest-lying single-soliton states and thermodynamically stable zero-soliton states can be much smaller than the magnetic Gibbs free energy of the latter states, provided that the intraband "penetration depths" (15) differ substantially and the interband coupling is weak. (In order to establish this important physical fact, we had to evaluate self-consistantly the vector potential. The results of this evaluation may be of interest in themselves.)

Our consideration encompasses in a natural way the case of superconducting Josephson-coupled bilayer structures studied experimentally in Ref 2.2 . Our conclusion that the self-energy of soliton states increases monotonically with an increase in the strength of interband coupling qualitatively agrees with the observations reported therein.

Furthermore, as a particular limit, our consideration contains the case of zero interband coupling. In view of the recently discussed possibility of independent superconductivity of electrons and protons in a liquid metallic state of hydrogen, ${ }^{21}$ some of our results may find application in this situation as well.

Finally, the exact soliton solutions derived in this paper should be compared with the exact soliton solutions representing equilibrium Josephson vortices in a superconducting tunnel junction. 17,19 In particular, Josephson vortices are pinned in their equilibrium positions owing to interaction with the edges of the junction. In contrast, in rotationally symmetric doubly-connected two-band superconductors, soliton positions are not fixed, which gives rise to a specific zero-frequency rotational mode. However, any defects that break rotational symmetry must cause soliton pinning. The effect of this pinning requires a separate discussion. In conclusion, we hope that our paper will stimulate further experimental and theoretical studies of the intriguing phenomenon of soliton states in two-band superconductors.

\section{Appendix A: Exact solution of the boundary-value problem for the vector potential}

The exact solution to (32) has the following form:

$$
\begin{gathered}
A(r)=\frac{r h_{O}}{2}, \quad r \in\left[0, r_{1}\right] \\
=-\frac{\Phi_{0}}{2 \pi r} q\left(n_{1}, n_{2}\right)+C_{1} I_{1}\left(\frac{r}{\lambda}\right)+C_{2} K_{1}\left(\frac{r}{\lambda}\right), \quad r \in\left(r_{1}, r_{2}\right] \\
h(r)=h_{O}, \quad r \in\left[0, r_{1}\right] \\
=\frac{C_{1}}{\lambda} I_{0}\left(\frac{r}{\lambda}\right)-\frac{C_{2}}{\lambda} K_{0}\left(\frac{r}{\lambda}\right), \quad r \in\left(r_{1}, r_{2}\right] \\
h_{O}=\frac{2 \lambda H f_{2}\left(\frac{r_{1}}{\lambda}, \frac{r_{1}}{\lambda}\right)}{r_{1} f_{1}\left(\frac{r_{2}}{\lambda}, \frac{r_{1}}{\lambda}\right)+2 \lambda f_{2}\left(\frac{r_{2}}{\lambda}, \frac{r_{1}}{\lambda}\right)}-\frac{\Phi_{0} q\left(n_{1}, n_{2}\right) f_{1}\left(\frac{r_{2}}{\lambda}, \frac{r_{1}}{\lambda}\right)}{\pi r_{1}\left[r_{1} f_{1}\left(\frac{r_{2}}{\lambda}, \frac{r_{1}}{\lambda}\right)+2 \lambda f_{2}\left(\frac{r_{2}}{\lambda}, \frac{r_{1}}{\lambda}\right)\right]} \\
C_{1} \equiv \frac{\lambda H\left[r_{1} K_{0}\left(\frac{r_{1}}{\lambda}\right)+2 \lambda K_{1}\left(\frac{r_{1}}{\lambda}\right)\right]}{r_{1} f_{1}\left(\frac{r_{2}}{\lambda}, \frac{r_{1}}{\lambda}\right)+2 \lambda f_{2}\left(\frac{r_{2}}{\lambda}, \frac{r_{1}}{\lambda}\right)}+\frac{\lambda \Phi_{0} q\left(n_{1}, n_{2}\right) K_{0}\left(\frac{r_{2}}{\lambda}\right)}{\pi r_{1}\left[r_{1} f_{1}\left(\frac{r_{2}}{\lambda}, \frac{r_{1}}{\lambda}\right)+2 \lambda f_{2}\left(\frac{r_{2}}{\lambda}, \frac{r_{1}}{\lambda}\right)\right]},
\end{gathered}
$$




$$
\begin{aligned}
C_{2} & \equiv \frac{\lambda H\left[r_{1} I_{0}\left(\frac{r_{1}}{\lambda}\right)-2 \lambda I_{1}\left(\frac{r_{1}}{\lambda}\right)\right]}{r_{1} f_{1}\left(\frac{r_{2}}{\lambda}, \frac{r_{1}}{\lambda}\right)+2 \lambda f_{2}\left(\frac{r_{2}}{\lambda}, \frac{r_{1}}{\lambda}\right)}+\frac{\lambda \Phi_{0} q\left(n_{1}, n_{2}\right) I_{0}\left(\frac{r_{2}}{\lambda}\right)}{\pi r_{1}\left[r_{1} f_{1}\left(\frac{r_{2}}{\lambda}, \frac{r_{1}}{\lambda}\right)+2 \lambda f_{2}\left(\frac{r_{2}}{\lambda}, \frac{r_{1}}{\lambda}\right)\right]} ; \\
f_{1}(x, y) & \equiv I_{0}(x) K_{0}(y)-I_{0}(y) K_{0}(x), \quad f_{2}(x, y) \equiv I_{0}(x) K_{1}(y)+I_{1}(y) K_{0}(x) .
\end{aligned}
$$

Here, $I_{\nu}(x)$ and $K_{\nu}(x)$ are modified Bessel functions of order $\nu=0,1 ; 18$ is the constant magnetic field in the opening. Expressions (A1)- (A3) are greatly simplified under condition (4):

$$
\begin{aligned}
& A(r)=\frac{r h_{O}}{2}, \quad r \in\left[0, r_{1}\right] ; \\
& =-\frac{\Phi_{0} q\left(n_{1}, n_{2}\right)}{2 \pi r} \frac{\sinh \frac{d}{\lambda}+\frac{2 \lambda}{r_{1}}\left(\cosh \frac{d}{\lambda}-\sqrt{\frac{r}{r_{1}}} \cosh \frac{r_{2}-r}{\lambda}\right)}{\sinh \frac{d}{\lambda}+\frac{2 \lambda}{r_{1}} \cosh \frac{d}{\lambda}} \\
& +\lambda H \sqrt{\frac{r_{2}}{r}} \frac{\cosh \frac{r-r_{1}}{\lambda}+\frac{2 \lambda}{r_{1}} \sinh \frac{r-r_{1}}{\lambda}}{\sinh \frac{d}{\lambda}+\frac{2 \lambda}{r_{1}} \cosh \frac{d}{\lambda}}, \quad r \in\left(r_{1}, r_{2}\right] \text {; } \\
& h(r)=h_{O}, \quad r \in\left[0, r_{1}\right] ; \\
& =-\frac{\Phi_{0} q\left(n_{1}, n_{2}\right)}{\pi r_{1} \sqrt{r r_{1}}} \frac{\sinh \frac{r_{2}-r}{\lambda}}{\sinh \frac{d}{\lambda}+\frac{2 \lambda}{r_{1}} \cosh \frac{d}{\lambda}} \\
& +H \sqrt{\frac{r_{2}}{r}} \frac{\sinh \frac{r-r_{1}}{\lambda}+\frac{2 \lambda}{r_{1}} \cosh \frac{r-r_{1}}{\lambda}}{\sinh \frac{d}{\lambda}+\frac{2 \lambda}{r_{1}} \cosh \frac{d}{\lambda}}, \quad r \in\left(r_{1}, r_{2}\right] ; \\
& h_{O}=\frac{-\frac{\Phi_{0} q\left(n_{1}, n_{2}\right)}{\pi r_{1}^{2}} \sinh \frac{d}{\lambda}+\frac{2 \lambda}{r_{1}} \sqrt{\frac{r_{2}}{r_{1}}} H}{\sinh \frac{d}{\lambda}+\frac{2 \lambda}{r_{1}} \cosh \frac{d}{\lambda}} .
\end{aligned}
$$

Expressions (A4)- (A6) should be compared with analogous expressions for a single-band-superconducting cylinder ${ }^{14}$ As can be easily seen, the cylinder exhibits a considerable Meissner effect under the conditions

$$
\frac{d}{\lambda} \ll 1, \quad \frac{d R}{2 \lambda^{2}} \gg 1
$$

In contrast, in the opposite case, when condition (5) is fulfilled, the Meissner effect is small, and expressions (A4)(A6) can be readily expanded up to first-order terms in $\varepsilon$. Taking into account a hierarchy of the small parameters of the problem,

$$
\begin{aligned}
\frac{d}{r_{1}} \approx \frac{d}{r_{2}} & \approx \frac{d}{R} \equiv 2 \varepsilon \frac{\lambda^{2}}{R^{2}}, \quad \frac{d}{\lambda} \equiv 2 \varepsilon \frac{\lambda}{R} ; \\
\frac{d}{R} & \ll \frac{d}{\lambda} \ll \varepsilon, \frac{\lambda}{R} \ll 1,
\end{aligned}
$$

we arrive at the first-order expressions (33) and (34).

$$
\text { Appendix B: A proof of the inequality }\left.G\left(|n| ; f_{H}\right)\right|_{|n|>1}>G\left(1 ; f_{H}\right)
$$

Consider the expression

$$
\begin{gathered}
\frac{2 \mathcal{L}_{m}}{\Phi_{0}^{2} \varepsilon} \Delta G\left(|n| ; f_{H}\right) \equiv \frac{2 \mathcal{L}_{m}}{\Phi_{0}^{2} \varepsilon}\left[G\left(|n| ; f_{H}\right)-G\left(0 ; f_{H}\right)\right] \\
=-\left|\theta\left(|n| c_{2}\right)\right|\left[2\left|\theta\left(f_{H}\right)\right|-\left|\theta\left(|n| c_{2}\right)\right|\right](1-\varepsilon)+\frac{4|n|^{2}}{\pi^{2}} c_{1} c_{2} K\left(k_{n}\right)\left[2 E\left(k_{n}\right)-\left(1-k_{n}^{2}\right) K\left(k_{n}\right)\right]
\end{gathered}
$$

that follows directly from (53). Our task is to prove that the right-hand side of (B1) for $|n|>1$ is larger than for $|n|=1$. Given that $c_{2} \in\left(0, \frac{1}{2}\right]$ by assumption (see the end of Section III), it is sufficient to provide a proof for 
$1<|n| \leq\left[\frac{1}{2 c_{2}}\right]+1$, where $\left[\frac{1}{2 c_{2}}\right]$ is the integer part of $\frac{1}{2 c_{2}}$. Indeed, the first term on the right-hand side of (B1) satisfies the inequality

$$
|| \theta\left(|n| c_{2}\right)\left|\left[2\left|\theta\left(f_{H}\right)\right|-\left|\theta\left(|n| c_{2}\right)\right|\right](1-\varepsilon)\right| \leq \frac{1}{4}(1-\varepsilon)
$$

whereas

$$
\frac{4 K\left(k_{n}\right)}{\pi^{2}}\left[2 E\left(k_{n}\right)-\left(1-k_{n}^{2}\right) K\left(k_{n}\right)\right] \geq 1
$$

Therefore, for $|n|>\left[\frac{1}{2 c_{2}}\right]+1$, when $|n| c_{2}>\frac{1}{2}$, any possible decrease in the first term on the right-hand side of (B1) due to an increase in $|n|$ cannot compensate for an incurred increase in the second term.

For $1<|n|<\left[\frac{1}{2 c_{2}}\right]+1$, there holds the relation $|n| c_{2} \leq \frac{1}{2}$, and (B1) becomes

$$
\begin{gathered}
\left.\frac{2 \mathcal{L}_{m}}{\Phi_{0}^{2} \varepsilon} \Delta G\left(|n| ; f_{H}\right)\right|_{|n|>1} \\
=|n| c_{2}\left[|n|\left(1-c_{2} \varepsilon\right)-2\left|\theta\left(f_{H}\right)\right|(1-\varepsilon)\right]+|n|^{2} c_{1} c_{2}\left[\frac{4 K\left(k_{n}\right)}{\pi^{2}}\left[2 E\left(k_{n}\right)-\left(1-k_{n}^{2}\right) K\left(k_{n}\right)\right]-1\right] \\
=|n| \frac{2 \mathcal{L}_{m}}{\Phi_{0}^{2} \varepsilon} \Delta G\left(1 ; f_{H}\right)+c_{2}|n|(|n|-1)\left[\left(1-c_{2} \varepsilon\right)+\frac{4 c_{1} K\left(k_{n}\right)}{\pi^{2}}\left[2 E\left(k_{n}\right)-\left(1-k_{n}^{2}\right) K\left(k_{n}\right)\right]-c_{1}\right] \\
>\frac{2 \mathcal{L}_{m}}{\Phi_{0}^{2} \varepsilon} \Delta G\left(1 ; f_{H}\right),
\end{gathered}
$$

which was to be proved.

\section{Appendix C: Particular examples of soliton solutions}

In Fig. 3. we present several different soliton solutions obtained numerically. (For greater clarity, we plot the derivatives $\frac{d \phi_{n}}{d \varphi}$.) However, in two limiting cases soliton solutions possess asymptotics in terms of elementary functions. Thus, for $\frac{R}{l} \ll 1$, we have: $\stackrel{17,19}{ }$

$$
\phi_{n}(\varphi) \approx \frac{(1+\operatorname{sgn} \gamma) \pi}{2}+n\left(\varphi-\varphi_{0}\right)+\frac{R^{2}}{n^{2} l^{2}} \sin \left[n\left(\varphi-\varphi_{0}\right)\right]
$$

The self-energy of soliton solutions (C1) is

$$
F_{\text {sol }}(n) \approx \frac{\Phi_{0}^{2} \varepsilon}{2 \mathcal{L}_{m}}|n|^{2} c_{1} c_{2}\left(1+\frac{2 R^{2}}{|n|^{2} l^{2}}\right)
$$

[Notice that expression (C2) clearly illustrates the general features of the self-energy of soliton solutions established in Section IV.]

In the opposite limiting case, when $1 \ll \frac{R}{l}<\infty$, asymptotics can be derived only for the single-soliton solutions $(|n|=1)$. Fixing the constants of integration by the condition $\varphi_{n 0}=\pi$, we get:

$$
\phi_{ \pm 1}(\varphi)=\frac{(1+\operatorname{sgn} \gamma) \pi}{2} \pm\left[-\pi+4 \arctan e^{\frac{R(\varphi-\pi)}{l}}+8 e^{-\frac{2 \pi R}{l}} \sinh \frac{R(\varphi-\pi)}{l}+o\left(e^{-\frac{2 \pi R}{l}}\right)\right] .
$$

The self-energy of these solutions is

$$
F_{\text {sol }}( \pm 1)=\frac{\Phi_{0}^{2} \varepsilon}{\mathcal{L}_{m}} \frac{4 R}{\pi l} c_{1} c_{2}\left[1+o\left(e^{-\frac{2 \pi R}{l}}\right)\right] .
$$

Solutions (C3) approach the well-known ${ }^{17}$ exact single-soliton solutions of the static sine-Gordon equation on an infinite interval:

$$
\phi_{ \pm 1}(\varphi)=\frac{(1+\operatorname{sgn} \gamma) \pi}{2} \pm\left[-\pi+4 \arctan e^{\pi x}\right], x \equiv \frac{R(\varphi-\pi)}{\pi l} \in(-\infty,+\infty)
$$



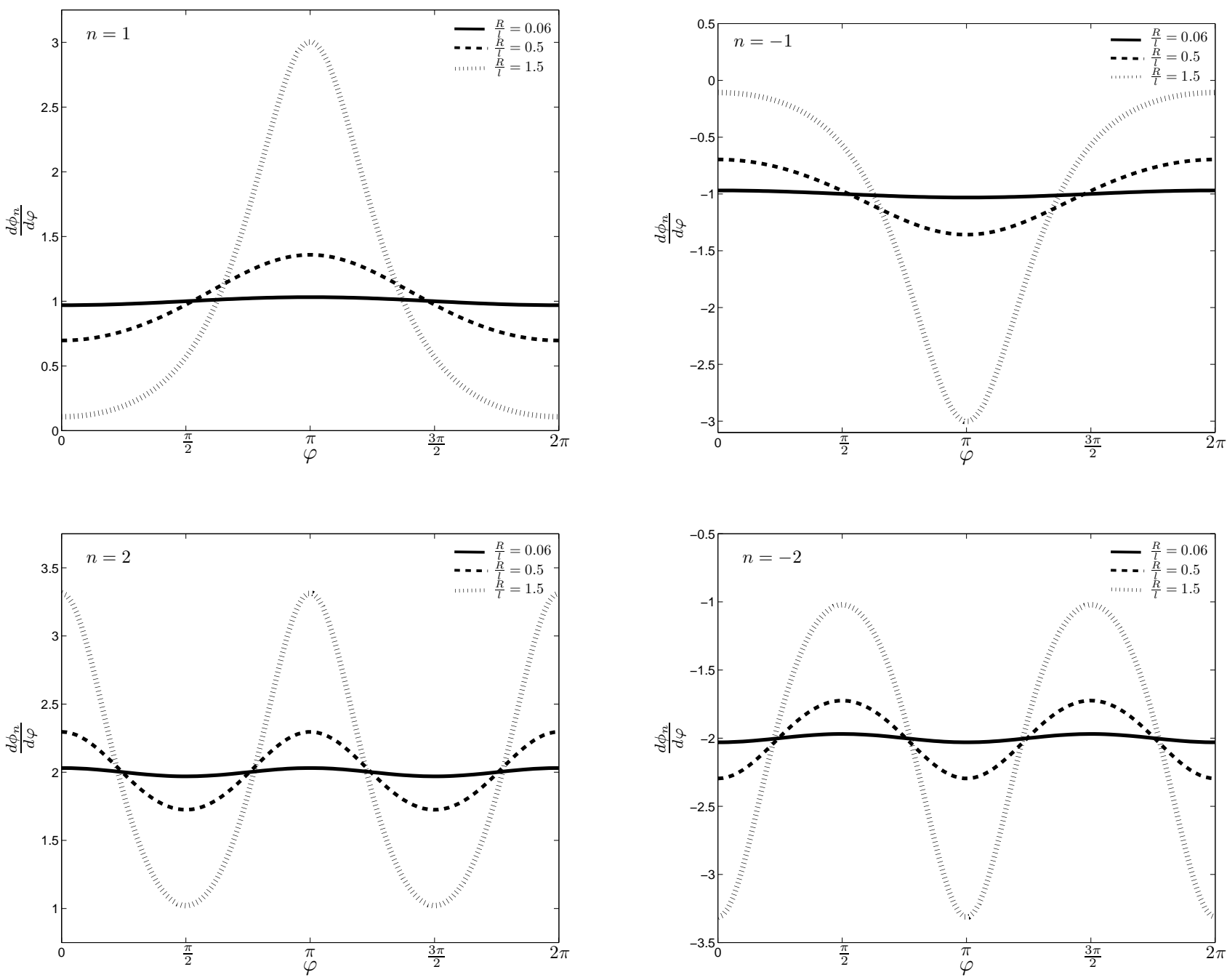

FIG. 3: Particular examples of soliton solutions $(n= \pm 1, \pm 2)$. The constants of integration in (46) are fixed by the condition $\varphi_{n 0}=\pi$.

* Electronic address: yerin@ilt.kharkov.ua

1 Y. Tanaka, Phys. Rev. Lett. 88, 017002 (2002).

${ }^{2}$ H. Bluhm, N. C. Koshnick, M. E. Huber, and K. A. Moler, Phys. Rev. Lett. 97, 237002 (2006); ibid. 98, 209902(E) (2007). It should be noted that the authors "mimicked" two-band superconductivity by using superconducting rings consisting of two parallel, Josephson-coupled aluminium layers. However, in this particular geometrical configuration, the composite system behaves like a genuine two-band superconductor: see the discussion of the Gibbs free-energy functional (8).

3 E. Babaev, Phys. Rev. Lett. 89, 067001 (2002); Nucl. Phys. B 686, 397 (2004).

${ }^{4}$ Y. Tanaka, A. Iyo, K. Tokiwa, T. Watanabe, A. Crisan, A. Sundaresan, and A. Terada, Physica C 470, S996 (2010); ibid. 1010 (2010).

5 See E. Mertzbacher, Am. J. Phys. 30, 237 (1962) for a clear discussion of this nontrivial condition in quantum physics.

6 The integers $n_{1}$ and $n_{2}$ are called "topological numbers" or "winding numbers": see, e.g., N. D. Mermin, Rev. Mod. Phys. 51, 591 (1979).

7 M. E. Zhitomirsky and V.-H. Dao, Phys. Rev. B 69, 054508 (2004).

8 A. Gurevich and V. M. Vinokur, Phys. Rev. Lett. 90, 047004 (2003); ibid. 97, 137003 (2006).

9 Y.S. Yerin and A.N. Omelyanchouk, Fiz. Nizk. Temp. 33, 538 (2007). 
10 Y.S. Yerin, S. V. Kuplevakhsky, and A.N. Omelyanchouk, Fiz. Nizk. Temp. 34, 1131 (2008).

11 J. Geyer, R. M. Fernandes, V. G. Kogan, and J. Schmalian, Phys. Rev. B 82, 104521 (2010).

12 See S. V. Kuplevakhsky and S. V. Naydenov, Phys. Rev. B 56, 2764 (1997) for a microscopic derivation of functionals of the type (8) in this particular case.

13 The same arguments apply to single-gap superconductors as well: see, e.g., P. G. de Gennes, Superconductivity of Metals and Alloys (Benjamin, New York, 1966).

14 V. L. Ginzburg, Zh. Eksp. Teor. Fiz. 42, 299 (1962).

15 I. N. Askerzade, Physics-Uspekhi 49, 1003 (2006).

16 See, e.g., L. D. Landau and E. M. Lifshitz, Electrodynamic of Continuous Media (Pergamon Press, Oxford, 1968). For reference purposes, we remark that the kinetic inductance is $\mathcal{L}_{k}=\mathcal{L}_{m} \frac{1-2 \varepsilon}{\varepsilon}$, as follows from relation (37). Accordingly, the expansion parameter can be expressed as $\varepsilon=\frac{\mathcal{L}_{m}}{\mathcal{L}_{k}+2 \mathcal{L}_{m}} \approx \frac{\mathcal{L}_{m}}{\mathcal{L}_{k}} \ll 1$.

17 S. V. Kuplevakhsky and A. M. Glukhov, Phys. Rev. B 73, 024513 (2006).

18 M. Abramowitz and I. A. Stegun, Handbook of Mathematical Functions (Dover, New York, 1965).

19 S. V. Kuplevakhsky and A. M. Glukhov, Phys. Rev. B 76, 174515 (2007); Fiz. Nizk. Temp. 36, 1253 (2010).

20 R. Jackiw, Rev. Mod. Phys. 49, 681 (1977).

21 E. Babaev, A. Sudbo, and N. W. Ashcroft, Nature (London) 431, 666 (2004). 